\title{
USOS MEDICINALES de los CÍTRICOS (CITRUS L., RUTACEAE) ENTRE LOS CRIOLlOS DEL SUR DE Misiones (ARGENTINA)
}

\author{
Pablo C. Stampella ${ }^{1,3 *}$, Norma I. HilgerT ${ }^{2,3}$ \& M. Lelia Pochettino ${ }^{1,3}$ \\ ${ }^{1}$ Laboratorio de Etnobotánica y Botánica Aplicada (LEBA), Facultad de Ciencias Naturales y Museo, Universidad Nacional de La Plata. \\ ${ }^{2}$ Instituto de Biología Subtropical (IBS), Facultad de Ciencias Forestales, Universidad Nacional de Misiones. \\ ${ }^{3}$ Consejo Nacional de Investigaciones Científicas y Técnicas - CONICET.
}

Recebido em 13 de agosto de 2015. Aceito em 13 fevereiro de 2017. Publicado em 23 abril de 2018.

\begin{abstract}
RESUmen - Los cítricos (Citrus L., Rutaceae) son plantas útiles introducidas hacia inicios del siglo XVI en diversos enclaves de América, con variedades cultivadas y espontáneas, especialmente en zonas tropicales y subtropicales. En poblaciones locales son considerados "plantas de la zona" presentando una alta diversidad de usos, entre ellos los medicinales. El objetivo de este trabajo es describir qué etnovariedades de cítricos son empleadas como medicinales por los criollos del sur de Misiones, como también las preparaciones, combinaciones con otras plantas y dolencias tratadas con las mismas. Se empleó la metodología etnobotánica cualitativa consistente en entrevistas semiestructuradas, caminatas con los informantes y observación participante, en 36 unidades domésticas (UD) de dos enclaves rurales del sur de Misiones (Argentina), durante los años 2010 y 2013. Se contó con el consentimiento informado previo de un adulto de cada UD. Se relevaron 7 etnoespecies de cítricos, prefiriéndose aquellas espontáneas, que pueden combinarse con otras plantas locales, para el tratamiento y prevención de 34 dolencias, principalmente relacionadas a estados gripales y febriles, hipertensión arterial, tos, trastornos nerviosos y digestivos. Las partes más empleadas fueron el jugo del endocarpo y las hojas, sin procesamiento y en infusiones y cocimientos. Teniendo en cuenta algunas particularidades, las etnoespecies son similares a las empleadas por comunidades de criollos en el norte de la provincia, representando importantes recursos alimentarios y medicinales.
\end{abstract}

Palabras clave: Alimentos funcionales; Cerro Mártires; Dolenclas; Espontáneas; Etnoespecies; Medicina tradicional; Teyú CUARÉ.

\section{Uso medicinal dos cítricos (Citrus L., Rutaceae) entre Criollos do sul de Misiones (Argentina)}

Resumo - Os cítricos (Citrus L., Rutaceae) são plantas úteis introduzidas desde o início do século XVI em vários enclaves na América, com variedades cultivadas e espontâneas, especialmente em zonas tropicais e subtropicais. Nas populações locais são consideradas "plantas da área", apresentando uma grande diversidade de usos, incluindo medicinal. O objetivo deste trabalho é descrever quais etnovariedades de cítricos são usadas como remédio pelos criollos nativos do sul de Misiones, bem como as formas de preparo, combinações com outras plantas e as doenças tratadas. Foi empregada a metodologia qualitativa da Etnobotânica, utilizado-se entrevistas semi-estruturadas, caminhadas com informantes e observação participante, em 36 unidades residenciais (UD) em dois enclaves rurais do sul de Misiones (Argentina), durante os anos de 2010 e 2013. Contamos com o consentimento prévio de um adulto de cada UD. Foram identificadas 7 etnoespécies de cítricos, sendo as espontâneas as preferidas, que podem ser combinadas com outras plantas locais para o tratamento e prevenção de 34 doenças, principalmente relacionados à gripe e estados febris, hipertensão, tosse, transtornos nervosos e digestivos. As partes mais utilizadas foram o suco do endocarpo e as folhas, sem transformação e infusões e cozimentos. Considerando-se algumas particularidades, as etnoespécies são similares às empregadas por comunidades criollas no norte da província, representando importantes recursos alimentares e medicinais. 
Palavras-chave: Alimentos Funcionais; Cerro Mártires; Doenças; Espontâneas; Etnoespécies; Medicina Tradicional; Teyú CUARÉ.

Medicinal uses of citrus (Citrus L., Rutaceae) among the Criollos of southern Misiones (Argentina)

Abstract - Citrus (Citrus L., Rutaceae) are useful plants introduced in the early sixteenth century in diverse enclaves of America, both cultivated and spontaneous varieties, especially in tropical and subtropical zones. The local populations consider them as "plants of the area" with high diversity of uses, including the medicinal. The aim of this article is to describe which ethno-varieties of citrus are used as remedy by the native creoles of the southern Misiones, as well as the methods of preparation, combinations with other plants and the diseases treated with them. Between 2010 and 2013, qualitative ethnobotanical methodology was applied in 36 residential units (DU) in two rural settlements of southern Misiones (Argentina), using semi-structured interviews, walks with informants and participant observation. Prior consent was obtained from one adult of each DU. Seven citric ethnospecies were identified, the spontaneous varieties were identified as the preferred ones, which can be combined with other local plants for the treatment and prevention of 34 diseases, mainly those related to influenza and fever, hypertension, cough, nervous and digestive disorders. The most common used parts were endocarp juice and leaves in infusions and baking without processing. The ethnospecies represent important food and medicinal resources and are similar to those used by creole communities in the north of the province, although showing local particularities.

Keywords: Functional Foods; Cerro Mártires; Diseases; Spontaneous; Ethnospecies; Traditional Medicine; Teyú Cuaré.

\section{INTRODUCCIÓN}

Los cítricos (Citrus L., Rutaceae, Aurantioideae) son originarios de las zonas cálidas del sur y sudeste de Asia hasta las zonas templadas de China, Australia e islas del Pacífico suroccidental (Gmitter \& Hu 1990). Su estrecha relación con las comunidades locales puede rastrearse en escritos chinos de más de 4000 años en los que son mencionados entre las plantas de los emperadores (Webber 1943). Luego de la caída del imperio Romano fueron dispersados por los árabes desde India hacia las costas del Mediterráneo ingresando por la península Ibérica a España durante los siglos X-XI (Ramón-Laca 2003). Su temprana introducción en América data del segundo viaje de Colón, y su arribo a la actual provincia de Misiones está relacionado con el establecimiento de las Misiones Jesuíticas de Guaraníes durante los siglos XVII-XVIII, temática desarrollada por Capparelli et al. (2011), Stampella et al. (2013) y Hilgert et al. (2014).

Este germoplasma ingresado al sur de la provincia, y en relación con las comunidades locales durante 5 siglos, actualmente es muy variable. En trabajos etnobotánicos recientes se han relevado 30 etnovariedades de cítricos agrupadas en 7 etnoespecies, correspondientes a 4 especies botánicas y 4 taxa híbridos según la sistemática actual (Capparelli et al. 2011). Entendiendo como etnoespecies a las "identidades folclóricas" reconocidas por las comunidades locales y, en la mayoría de los casos, referidas mediante un nombre vernáculo, según lo propuesto por Zamudio \& Hilgert (2012). Resultados similares, aunque con una menor variabilidad, se han encontrado para la zona de las Misiones Jesuíticas de Baja California (De Grenade et al. 2014). Esta estrecha relación entre comunidades locales y cítricos a través de un considerable período temporal resultó en la resignificación de este género botánico y en el empleo de los mismos en la planificación del paisaje local volviéndose, de esta manera, elementos conspicuos en diversos ambientes de las unidades domésticas (UD). Así, estas variedades locales, cultivadas y espontáneas, no son percibidas como plantas exóticas o extrañas, sino más bien como plantas que conforman el paisaje cotidiano, legado de las generaciones pasadas (Stampella et al. 2013). 
Además de su uso como frutal y ornamental los cítricos son ampliamente empleados como medicinales. Arias \& Ramón-Laca (2005) citan diversos usos relevados en la bibliografía histórica de la Antigüedad y Edad Media, y registran una mayor diversidad de usos medicinales pasados que actuales. Para Argentina -y seguramente Paraguay, Brasil y Bolivia- al indagar en las obras de plantas medicinales populares, inventarios de mercados y trabajos etnobotánicos en diversas comunidades del nordeste argentino (NEA), puede apreciarse una alta diversidad de cítricos empleados actualmente para distintas dolencias (Stampella et al. 2014).

Hieronymus (1882) incluye a los cítricos más conocidos en su Plantae diaphoricae documentando varias enfermedades relacionadas principalmente con el sistema digestivo, además de su uso como antiescorbútico, y contra parásitos y venenos. Asimismo, el trabajo de Martínez-Crovetto (1981) acerca de la medicina popular del NO de Corrientes se explaya en detalles acerca de las etnoespecies más conocidas como el "naranjo agrio", "limonero", "pomelo", "mandarina” y "naranjo". Entre los guaraníes de territorio paraguayo, Bertoni (1927) cita a la "lima sutî" [Citrus $\times$ aurantiifolia (Christm.) Swingle] para el tratamiento de enfermedades epidémicas como malaria y paludismo, comunes en la zona debido a la abundancia de vectores. Desde mediados del siglo pasado aparecen en Paraguay obras de plantas medicinales, en las que se recopilan los usos populares de la flora local, y sus nombres combinan la lengua guaraní con la castellana; en estos textos abundan las menciones de los cítricos (Cadogan 1957; Burgstaller 1985; Rodríguez Barboza 1985).

Para Misiones se pueden destacar diversos trabajos sobre plantas medicinales, algunos de los cuales comparan su aplicación entre distintos grupos culturales. Amat \& Yajía (1991) presentan un inventario de las plantas vendidas en el mercado de Posadas; citan entre los cítricos el "apepú" contra la hipertensión y para enfermedades respiratorias. Crivos et al. (2007) mencionan el uso de las cáscaras de "mandarina" (Citrus reticulata Blanco) en comunidades M’bya para el tratamiento de la "rotación del estómago", dolencia relacionada al sistema digestivo. Pero es con los trabajos etnobotánicos y etnobiológicos entre los "criollos" y colonos, donde los cítricos presentan valores de uso comparables a las plantas nativas. En los alrededores de la reserva Yabotí (E de Misiones), Keller \& Romero (2006) relevaron los usos medicinales de las plantas entre los "criollos", presentando los cítricos frecuencias de uso similares a plantas nativas como "cedrón del campo" [Aloysia gratissima (Gillies y Hook) Tronc.], “cocú” [Allophyllus edulis (A. St.-Hil., A. Juss. y Cambess.) Hieron. ex Niederl.], "cedrillo" (Guarea macropbylla Vahl), "ambay" (Cecropia pachystachya Trécul) y "lapacho negro" [Handroanthus heptaphyllus (Vell.) Mattos]. Esta importancia puede verse, asimismo, en los trabajos de Zamudio et al. (2010) y Zamudio \& Hilgert (2011), donde se explora el empleo de remedios compuestos a base de mieles de Apidae por los "criollos" y "polacos" del norte de la provincia. En los mismos, los cítricos se encuentran entre las plantas más mencionadas junto a "eucalipto" (Eucalyptus sp.), "ambay” y “ajo" (Allium sativum L.). En las investigaciones de Kujawska et al. (2012), Kujawska \& Hilgert (2014) y Kujawska \& Pieroni (2015) los cítricos se encuentran entre las plantas más empleadas por los polacos y criollos misioneros del norte de la provincia, donde los cítricos (especialmente, la naranja y el limón mandarina) presentan los valores más altos de menciones.

Las propiedades medicinales de los cítricos también han sido estudiadas desde un enfoque etnobotánico histórico y actual en el sur de la provincia por Stampella et al. (2013). En este trabajo se releva sus usos medicinales locales comparándolos con los usos antiguos y con la búsqueda de compuestos activos medicinales. Asimismo, se destaca la diversidad relevada y los distintos espacios (microambientes) de la UD donde son cultivados o permanecen como espontáneos.

Dada la importancia de este género botánico introducido tempranamente, apropiado por las comunidades 
locales de Misiones, y a la vez combatido en algunos Parques Nacionales de Argentina y Brasil (Rodolfo et al. 2008) cabe preguntarse qué etnovariedades son empleadas como medicinales y qué afecciones se tratan con ellas, así como la similitud o divergencia del elenco de etnovariedades empleadas con respecto a otros enclaves de la provincia.

En consecuencia, el objetivo de este trabajo es describir qué etnovariedades de cítricos son empleadas como medicinales por los criollos del sur de Misiones, como también las preparaciones, combinaciones con otras plantas y dolencias tratadas con las mismas.

\section{Materiales y MÉTODOS}

Área de Estudio

El trabajo de campo fue realizado en los departamentos de San Ignacio y Concepción de la Sierra (Misiones, Argentina) focalizándose principalmente las localidades rurales de Teyú Cuaré y paraje Cerro Mártires, respectivamente. Sin embargo, se ha trabajado también con informantes de enclaves urbanos y periurbanos, como las ciudades de San Ignacio y Concepción, el poblado de La Corita (Concepción) y el asentamiento de Puerto Viejo (San Ignacio) (Figura 1).

Figura 1. Área de estudio. Se indica la provincia de Misiones y, en la misma, los sitios estudiados (Teyú Cuaré y Cerro Mártires) con imagen satelital ampliada (Google Earth).

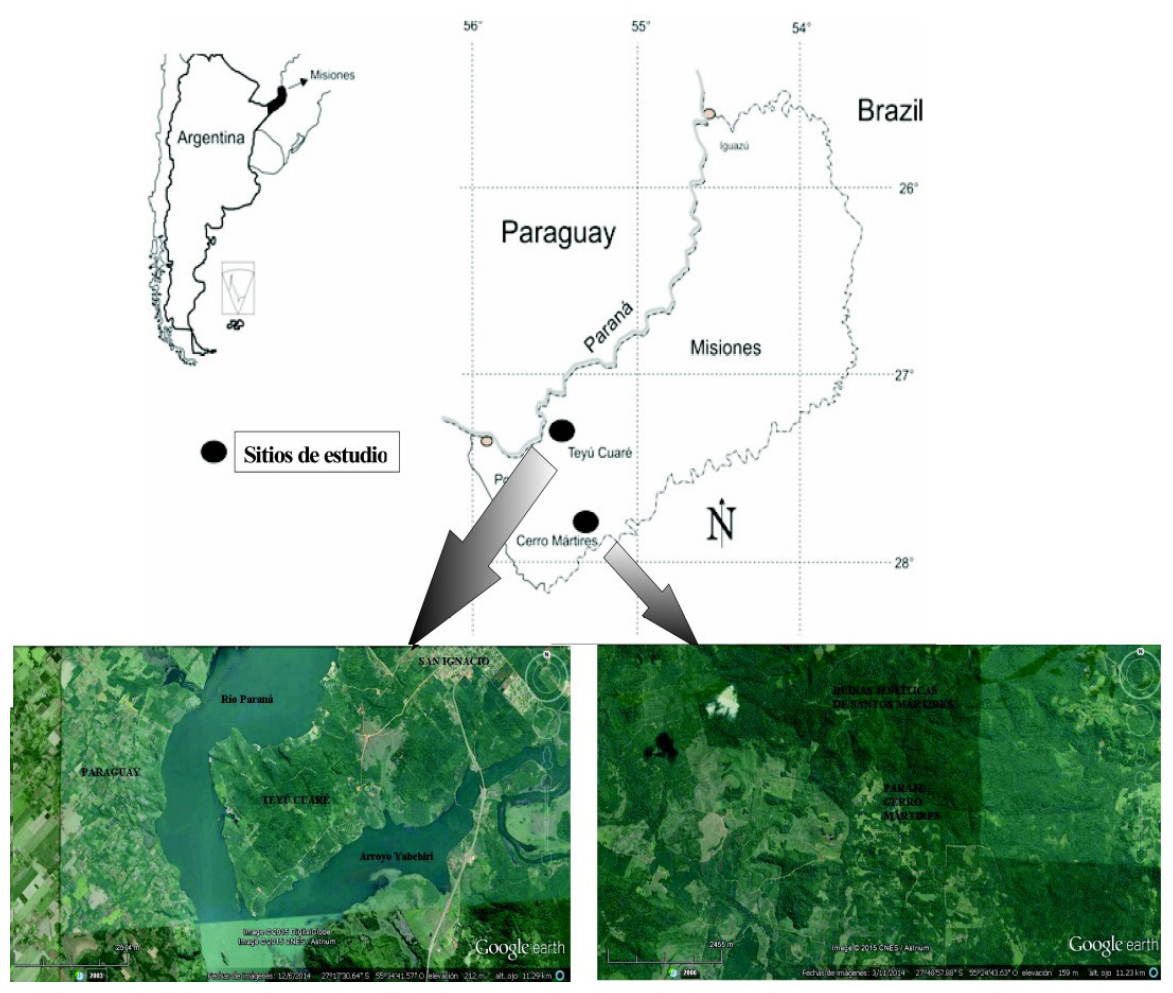

En los ámbitos urbanos y periurbanos la mayoría de los entrevistados residen en casas de material o de madera, en terrenos de entre 600 y $2500 \mathrm{~m}^{2}$, se desempeñan en diversas profesiones y algunos son jubilados o amas de casa. En esta situación de urbanismo los espacios verdes familiares dispuestos alrededor del hogar 
se destinan en distintas proporciones a jardines (áreas de cultivo de plantas ornamentales) y pequeñas buertas (espacios para la producción de verduras); estos espacios son algo mayores en zonas periurbanas, donde se cultiva mandioca, maíz, porotos, batatas y algunos frutales. En cambio, en los ámbitos rurales la principal actividad de la mayoría de los informantes es la horticultura (chacreros) o el trabajo en la casa (que incluye la actividad antedicha). Sin embargo, realizan distintos trabajos temporales en empresas forestales, yerbateras y tealeras. Sus viviendas, generalmente son de madera -recientemente con techos de chapa de zinc- con la adición -en algunos casos- de construcciones de mampostería. En prácticamente todos los casos la superficie de los lotes es de 25 hectáreas, mientras que la destinada al cultivo y cría de ganado varía en entre 5 y 15 hectáreas. En ambas cuencas, los enclaves rurales están conformados por caseríos dispersos, agrupados relativamente próximos a las escuelas primarias. Cuando los rozados (terrenos desmontados para la producción agrícola) en uso se encuentran alejados de las viviendas, en su proximidad se construyen casas temporales las que, luego de abandonadas, devienen en taperas (término de uso común en Argentina para las viviendas desocupadas o abandonadas). Dependiendo del acceso a la tierra los campesinos se apropian del entorno mediante la estrategia de uso múltiple, con una economía por lo general de subsistencia en la que se combinan prácticas de roza y quema, horticultura y sistemas agroforestales a pequeña escala, ganadería, caza y recolección en algunos sectores (Stampella et al. 2013).

En los sitios tratados, se observaron escasas UD que venden su cosecha y productos elaborados (conservas, dulces, mermeladas, derivados lácteos) en las ferias francas de San Ignacio y Concepción. En la mayoría de los casos corresponden a familias descendientes de colonos ucranianos, polacos y alemanes. Para el caso de Cerro Mártires las características de las fincas y de los pobladores coinciden con las descriptas por Granitto y Sarandón (2002) para el paraje de San Juan de la Sierra, situado a 10 kilómetros al O-NO, donde predominan las prácticas agrosilvícolas de subsistencia. Así, los productores con grandes extensiones de tierra tienden a simplificar los sistemas productivos con monocultivos, mientras que las familias con superficies intermedias (25 hectáreas) se caracterizan por la producción por subsistencia y eventual venta de los excedentes.

Los enclaves estudiados son muy diversos culturalmente, conformados por convivencia -a veces incluso en una misma UD- de criollos (descendientes de los primeros colonizadores y guaraníes), "paisanos" (M’bya), y colonos o polacos (descendientes de los inmigrantes de principios de siglo XX). Sin embargo de acuerdo a la cercanía con las fronteras de los países vecinos, el grupo mayoritario de los "criollos" se relaciona a ambos países, hablando guaraní o portugués (o mezclas con español, denominados localmente guarañol y portuñol, respectivamente). Además, hay una fuerte presencia de inmigrantes provenientes de los vecinos países de Paraguay y Brasil (sur) que se establecieron de modo informal e independiente a lo largo de los dos últimos siglos (Stampella et al. 2013); situación frecuentemente citada en diferentes enclaves de la provincia (Keller \& Romero, 2006; Zamudio et al. 2010).

$\mathrm{Al}$ inicio del trabajo de campo se obtuvo el consentimiento previamente informado de un adulto de cada UD involucrado en la investigación. Para ello se explicó brevemente, en lenguaje apropiado y divulgativo, los objetivos, fundamentación, implicancias y alcances de la investigación (Alexiades \& Laird 2002; Lewis 2010) acordando con los participantes distintos ítems de consentimiento.

Se trabajó con 36 UD cuyos informantes variaron en edades de entre 18 y 88 años, durante el período comprendido entre 2010 y 2013. Las UD fueron seleccionadas aleatoriamente en un principio y luego por método de bola de nieve. Se empleó la metodología etnobotánica cualitativa consistente en observación participante, entrevistas semiestructuradas, y caminatas con los informantes por los diversos espacios de la UD (Cotton 
1998; Albuquerque et al. 2014). Durante las mismas se indagó acerca de las dolencias tratadas con los cítricos, etnoespecies y etnovariedades empleadas, partes empleadas, formas de preparación, de administración, y otras plantas y compuestos empleados en la elaboración de los preparados, obteniendo apreciaciones locales de la diversidad y las prácticas a partir del discurso.

El material de referencia, consistente en partes de plantas fértiles y estériles, fue colectado en compañía de los informantes, acondicionado, identificado y depositado en los herbarios del Museo de La Plata (LP) y del Laboratorio de Etnobotánica y Botánica Aplicada de la Facultad de Ciencias Naturales y Museo de la Universidad Nacional de La Plata.

\section{REsultados}

\section{Las etnoespecies empleadas}

Los cítricos están representados en todas las UD visitadas. En los enclaves urbanos son comunes en los jardines y huertas, aún en aquellos de pequeñas dimensiones, donde son cultivados principalmente con fines ornamentales, como frutales y medicinales. Hacia los enclaves periurbanos y rurales la superficie de los terrenos se incrementa, como también la abundancia y diversidad de cítricos y otros frutales, debido al aprovechamiento de las etnovariedades espontáneas. En estos enclaves, los microambientes de los terrenos incluyen, además de jardines y huertas, montes frutales (huertos frutales), espacios destinados a la cría de ganado (campos), al cultivo de plantas anuales (rozados), como también otras parcelas en distintos estados de sucesión ecológica, también productivas (capueras). Asimismo, se encuentran presentes en los montes (áreas de vegetación supuestamente nativa, aunque puede ser selva secundaria) donde abundan especialmente en aquellos peridomiciliarios y sujetos a actividades agrosilvícolas, como la cría de ganado durante la época invernal.

Para la atención primaria de la salud son empleadas 7 etnoespecies de cítricos correspondientes a 5 taxa (dos taxa biológicos y tres taxa híbridos) según el tratamiento sistemático actual del género Citrus (Tabla 1).

Tabla 1 - Etnoespecies de cítricos empleadas como medicinales en los enclaves estudiados.

\begin{tabular}{llc}
\hline \multicolumn{1}{c}{ Etnoespecie } & \multicolumn{1}{c}{ Nombre específico } & \% de menciones \\
\hline Limón & Citrus $\times$ taitensis Risso & 23,9 \\
NARANJA & Citrus $\times$ aurantium L. [Grupo naranja dulce] cv 'Común' & 19,1 \\
ApEPú O NARANJA AMARGA & Citrus $\times$ aurantium L. [Grupo naranja amarga] cv. 'Apepú' & 17,7 \\
POMELO & Citrus $\times$ aurantium L. [Grupo pomelo] cv. 'Duncan' & 15,3 \\
LIMA & Citrus $\times$ limon (L.) Osb. cv. 'Lima de Persia' & 14,8 \\
MANDARINA & Citrus reticulata Blanco cv 'Común de Concordia' & 8,6 \\
CIDRA & Citrus maxima $($ Burm.) Merr. & 0,5 \\
\hline
\end{tabular}

Estas menciones se refieren principalmente a variedades propagadas mediante semillas -denominadas localmente comunes- diferenciadas en caseras y de monte, de acuerdo a los microambientes donde se encuentran. Esta denominación forma parte de la clasificación local de los cítricos que consiste en 3 grupos de etnovariedades: comunes, injertados y de injerto. Las etnovariedades comunes son aquellas propagadas mediante semillas en el ámbito doméstico, pudiendo ser a su vez caseras (cuando se cultivan en los microambientes domésticos) o de monte 
(espontáneas en las selvas). Las etnovariedades injertadas son aquellas variedades seleccionadas y comerciales que son multiplicadas vegetativamente mediante la técnica de injerto en un portainjerto determinado. Finalmente, las etnovariedades de injerto son aquellas empleadas en las plantaciones comerciales como portainjertos (Stampella et al. 2013). Las variedades caseras son cultivadas en los microambientes domésticos (jardín, huerta, monte frutal, campo y rozado), mientras que las variedades de monte son espontáneas en el monte o capueras, microambientes menos intervenidos. No obstante son preferidas estas últimas argumentando que "tienen más remedio".

Las combinaciones con otras plantas son también frecuentes (15\% de las menciones), generalmente en dolencias relacionadas a estados gripales, febriles y tos. Así, las preparaciones involucran hojas de "durazno" [Prunus persica (L.) Batsch.], brotes de "níspero" [Eriobotrya japonica (Thunb.) Lindl.], hojas y frutos de "eucalipto" (Eucalyptus spp.), ramas de "catai" [Persicaria punctata (Elliot.) Small], brotes y hojas de "ambay" (C. pachystachya), brotes de "talera" (Celtis sp.), hojas de "salvia" (Lantana sp.), hojas de "prontoalivio" o "salvia" [Lippia alba (Mill.) N.E.Br. ex Britton \& P.Wilson], hojas de "sota caballos" o "soita" (Luehea divaricata Mart.), ramas de "malva" (Malvaceae indet.), y ramas de "poleo", “cedrón del campo" o "niño rupá” [A.gratissima y A. virgata (Ruiz \& Pav.) Juss.]. A varias de estas plantas se les atribuyen propiedades analgésicas y diaforéticas empleadas de esta manera para sobrellevar los síntomas de estos padecimientos. Asimismo, el elenco de plantas varía según la época del año. Durante el verano no se encuentran disponibles las partes relativas a los frutos de los cítricos, empleándose entonces otras plantas locales. También se ha relevado el empleo conjunto de "pastillas" o "medicamentos" nominados según su marca comercial tratándose mayoritariamente de analgésicos, antiinflamatorios y antitérmicos elaborados con ácido acetil-salićlico, paracetamol, metamizol o dipirona, e ibuprofeno.

\section{Las dolencias tratadas}

Se relevaron 34 dolencias (emic, locales) tratadas con los cítricos (Tabla 2) siendo las más mencionadas aquellas referidas en la Figura 2A. Así, la mayoría de las menciones se corresponden a estados gripales y febriles, padecimientos respiratorios, digestivos, sanguíneos y nerviosos.

\section{Partes de la planta utilizada, preparaciones y formas de administración}

Entre las partes empleadas se incluyen el jugo del endocarpo (jugo del gomo), las hojas y los frutos enteros (fruta). Menos frecuentemente fueron mencionados el epicarpo (pie), las flores, el mesocarpo (pelusa) y los brotes (brotos) (Figura 2B, Tabla 2). Sin embargo, hay particularidades para cada una de las etnoespecies. La mandarina y la naranja son empleadas principalmente por sus hojas, mientras que el limón lo es por el jugo del endocarpo.

La forma de preparación de los remedios también es variable, siendo las más frecuentes el empleo directo del jugo del endocarpo (gomo) -puro o diluido en agua-, las infusiones y cocimientos de las partes antes mencionadas. También son empleados el calentamiento de los frutos enteros al rescoldo y asado de los mismos, la elaboración de jarabe y melitus, mate, tereré, preparación en dulces y freído en aceite vegetal (Figura 2C, Cuadro 1). Tanto el jarabe como el melitus son preparados con el jugo del endocarpo calentado y diluidos en miel de caña y miel de abejas, respectivamente. 


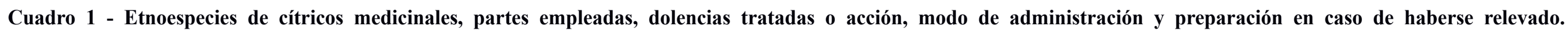

\begin{tabular}{|c|c|c|c|c|}
\hline Etnoespecie & Parte empleada & Propriedad atribuida & Modo de administración & Preparación de la receta \\
\hline CIDRA & Jugo & Preventivo & Jugo & \\
\hline \multirow{15}{*}{ LIMA } & \multirow{7}{*}{ Jugo } & Gripe & \multirow{7}{*}{ Jugo } & En el agua de la pava para cebar mate \\
\hline & & Para bajar la presión & & Bebido, frío o caliente \\
\hline & & Para el hígado & & Bebido \\
\hline & & Diabetes & & \\
\hline & & Digestivo & & \\
\hline & & Resfrío & & \\
\hline & & Tranquilizante & & \\
\hline & Fruto & Para bajar la presión & Cocimiento & Bebido, frío o caliente \\
\hline & Flores & Para el corazón & Infusión o mate & \\
\hline & \multirow{6}{*}{ Hojas } & Tranquilizante & \multirow{2}{*}{ Infusión } & Tomar por la mañana y por la noche \\
\hline & & Para el corazón & & 3 hojas por taza \\
\hline & & Diabetes & & \\
\hline & & \multirow{3}{*}{ Para bajar la presión } & Infusión & 3 hojas por taza, tomar por la mañana y por la noche \\
\hline & & & Macerado en frío & Hojas machacadas en agua fría. Tomar como agua o en tereré \\
\hline & & & Cocimiento & $\begin{array}{l}4 \text { ó } 5 \text { hojas en } 1 \text { litro de agua. Enfriar y tomar como agua durante el día. } \\
\text { También en caliente. }\end{array}$ \\
\hline \multirow{10}{*}{$\begin{array}{l}\text { POMELO BLANCO } \\
\text { Y ROSADO }\end{array}$} & \multirow{5}{*}{ Hojas } & \multirow{2}{*}{ Gripe } & Infusión & $\begin{array}{l}\text { Con } 2 \text { hojas, } 2 \text { de naranja, } 2 \text { de durazno, } 2 \text { de níspero y ambay. Tomar con } \\
\text { media pastilla }\end{array}$ \\
\hline & & & Cocimiento & $\begin{array}{l}\text { Con azúcar quemado y mezclado con brotes de talera. Se acompaña con una } \\
\text { novalgina o bayer }\end{array}$ \\
\hline & & Fiebre & Infusión & $\begin{array}{l}\text { Con } 2 \text { hojas, } 2 \text { de naranja, } 2 \text { de durazno, } 2 \text { de níspero y ambay. Tomar con } \\
\text { media pastilla }\end{array}$ \\
\hline & & Para la tos & Cocimiento & $\begin{array}{l}\text { Con azúcar quemado y mezclado con brotes de talera. Se acompaña con una } \\
\text { novalgina o bayer }\end{array}$ \\
\hline & & Para bajar la presión & Infusión & \\
\hline & \multirow{5}{*}{ Jugo } & Refrescante & \multirow{3}{*}{ Jugo } & Diluido en agua y bebido. También cebado en tereré. Quita la sed de la fiebre \\
\hline & & Preventivo & & "Es bueno para todo" \\
\hline & & Para la tos & & Agregado a infusiones de cáscaras de naranja y hojas de salvia \\
\hline & & Asma & \multirow{2}{*}{ Jugo calentado } & \multirow{2}{*}{ Bebido a cucharadas } \\
\hline & & Catarro & & \\
\hline
\end{tabular}




\begin{tabular}{|c|c|c|c|c|}
\hline \multirow{7}{*}{$\begin{array}{l}\text { POMELO BLANCO } \\
\text { Y ROSADO }\end{array}$} & \multirow{4}{*}{ Jugo } & Digestivo & \multirow{3}{*}{ Jugo } & Bebido \\
\hline & & Para bajar la presión & & "Es mejor el pomelo rosado que el blanco" \\
\hline & & \multirow{2}{*}{ Gripe } & & Tomado como agua \\
\hline & & & Jugo calentado & Bebido a cucharadas \\
\hline & Cáscara & Fiebre & Infusión & Con te negro, azúcar quemado, jugo de limón y geniol \\
\hline & \multirow{2}{*}{ Fruto } & Gripe & \multirow{2}{*}{$\begin{array}{l}\text { Como recipiente para } \\
\text { mate }\end{array}$} & \multirow{2}{*}{$\begin{array}{l}\text { Cortar la parte superior del fruto y ahuecarlo parcialmente. Rellenar con } \\
\text { yerba mate. Cebar. De vez en cuando apretarlo para que largue jugo }\end{array}$} \\
\hline & & Preventivo & & \\
\hline \multirow{18}{*}{ MANDARINA } & \multirow{3}{*}{ Jugo } & Refrescante & \multirow{3}{*}{ Jugo } & Consumidos durante las expediciones al monte \\
\hline & & Sedante & & \multirow{2}{*}{ Bebido } \\
\hline & & Digestivo & & \\
\hline & Brotes & Gripe & \multirow{3}{*}{ Infusión } & \\
\hline & \multirow{14}{*}{ Hojas } & \multirow{2}{*}{ Sedante } & & 2-4 hojas por taza. Tomar por la noche \\
\hline & & & & 3 hojas por taza. Tomar por la noche \\
\hline & & \multirow{2}{*}{ Fiebre } & Cocimiento & De 6-7 hojas \\
\hline & & & Infusión & \\
\hline & & Para la tos & \multirow{4}{*}{ cocimiento } & $\begin{array}{l}\text { Con azúcar quemado y mezclado con brotes de talera. Se acompaña con una } \\
\text { novalgina o bayer }\end{array}$ \\
\hline & & Antiséptico & & De 200 gramos de hojas, dejar enfriar \\
\hline & & Dolor de dientes & & Dejar enfriar y hacer buches \\
\hline & & Dolores de huesos & & Baños \\
\hline & & Para vomitar & \multirow{3}{*}{ Infusión } & De un puñado de hojas, tomar caliente \\
\hline & & \multirow{5}{*}{ Gripe } & & Con hojas de limón y ambay \\
\hline & & & & $\begin{array}{l}2 \text { hojas, } 2 \text { hojas de naranja, } 2 \text { hojas de salvia o prontoalivio. Agregar azúcar } \\
\text { quemado con un carboncito }\end{array}$ \\
\hline & & & Cocimiento & $\begin{array}{l}\text { Con azúcar quemado y mezclado con brotes de talera. Se acompaña con } \\
\text { novalgina o bayer }\end{array}$ \\
\hline & & & & De 5 hojas por taza de agua. Tomar con pastillas de medicamento \\
\hline & & & Infusión & De las hojas verdes trituradas. Endulzar con miel \\
\hline \multirow{4}{*}{ APEPÚ } & \multirow{4}{*}{ Jugo } & Refrescante & \multirow{3}{*}{ Jugo } & Diluido en agua y bebido. También cebado en tereré \\
\hline & & Preventivo & & Bebido \\
\hline & & Gripe & & Agregado a la infusión de te negro con aspirina \\
\hline & & & Cocimiento & $\begin{array}{l}\text { Del jugo de } 3 \text { frutos en } 1 \text { taza de agua, con miel de abejas y hojas de } \\
\text { eucalipto }\end{array}$ \\
\hline
\end{tabular}




\begin{tabular}{|c|c|c|c|c|}
\hline & & $\begin{array}{l}\text { Enfermedades } \\
\text { respiratorias }\end{array}$ & & Se mezcla el jugo con azúcar o miel y se entibia. Se bebe a cucharaditas \\
\hline & & Broncoespasmos & Jarabe & $\begin{array}{l}\text { Hervir } 3 \text { litros del jugo con } 1 \text { litro de miel hasta reducir a 1,25 o 1,5 litros. } \\
\text { Agregar canela y estacionar }\end{array}$ \\
\hline & & Para la tos & & Mezclado con miel. Tomar por la mañana en ayunas y por la noche \\
\hline & & & & Mezclado con azúcar. Beber caliente \\
\hline & & Angina & & Mezclado con 3 cucharadas de miel de abejas de monte \\
\hline & & Pasmo & Cocimiento & Con agregado de sal \\
\hline & & Para bajar la presión & & 4 hojas por taza \\
\hline & & Digestivo & Intusion & \\
\hline & & Dolor de huesos & Cocimiento & Baños en las zonas afectadas \\
\hline & & Gripe & Infusión & $\begin{array}{l}\text { Agregado en infusiones con te negro, cafiaspirina y azúcar quemada con un } \\
\text { carboncito }\end{array}$ \\
\hline & & & & En caña o cayaza. Enfriar y estacionar \\
\hline & & Angina & Cocimiento & Dejar enfriar y hacer gárgaras \\
\hline A PEPÍ & Hoia & Para la tos & Infusión & Junto a hojas de sota caballos \\
\hline АРЕРО & & Pasmo & & Aplicar en la zona afectada \\
\hline & & Pasmo & Cocimiento & Junto a hojas de malva y durazno. Realizar baños en la zona afectada \\
\hline & & Pasmo de columna & & $\begin{array}{l}\text { Junto a hojas de eucalipto y poleo. Realizar baños todos los días durante } 1 \\
\text { semana }\end{array}$ \\
\hline & & Dolor de panza & Infusión & \\
\hline & & Hemorroides & Cocimiento & $\begin{array}{l}\text { De } 20-30 \text { hojas, con } 2-3 \text { raíces de catai y una planta de malva blanca. Dejar } \\
\text { enfriar y hacer baños de asiento }\end{array}$ \\
\hline & & Digestivo & & 3 hojas por taza. Tomar en ayunas \\
\hline & & Fiebre & Infusión & Con te negro, azúcar quemado, jugo de limón y un geniol \\
\hline & Cáscara & Digestivo & & \\
\hline & & Piojicida & Jabón & Elaboración de jabones con aceite de coco \\
\hline & Mesocarpo & Digestivo & Dulce de oreja & $\begin{array}{l}\text { Los frutos previamente pelados (extracción del epicarpo) se cortan } \\
\text { longitudinalmente en } 4 \text {, se les extrae el gomo (endocarpo) y se dejan en una } \\
\text { corriente de agua para que se les vaya el amargor. Luego se hierven en miel de } \\
\text { caña durante algunas horas y se envasan. Se les puede agregar clavo de olor o } \\
\text { canela. }\end{array}$ \\
\hline
\end{tabular}




\begin{tabular}{|c|c|c|c|c|}
\hline \multirow{3}{*}{ APEPÚ } & \multirow{3}{*}{ Fruto } & Llagas de la aftosa & Medio fruto con sal & $\begin{array}{l}\text { Cortados longitudinalmente se les agrega un poco de sal y se raspan en la } \\
\text { lengua de la vaca. Luego se tira obliga, mediante un tironeo de la lengua, a } \\
\text { que lo trague }\end{array}$ \\
\hline & & Gripe & \multirow{2}{*}{ Asado o calentado } & \multirow{2}{*}{ Fruto asado al rescoldo. Beber su jugo tibio } \\
\hline & & Fiebre & & \\
\hline \multirow{21}{*}{ NARANJA } & \multirow{20}{*}{ Hoja } & Bajar la presión & \multirow{2}{*}{ Infusión } & \\
\hline & & \multirow{3}{*}{ Para la tos } & & De 2-5 hojas. Dejar entibiar y tomar por la noche \\
\hline & & & \multirow[t]{2}{*}{ Cocimiento } & $\begin{array}{l}\text { Con azúcar quemado y mezclado con brotes de talera. Se acompaña con una } \\
\text { novalgina o bayer }\end{array}$ \\
\hline & & & & Con hojas de salvia y/o de ambay. Agregar azúcar y tomar con una aspirina \\
\hline & & \multirow{3}{*}{ Fiebre } & \multirow[b]{2}{*}{ Infusión } & De 4-5 hojas. Dejar entibiar y tomar por la noche \\
\hline & & & & $\begin{array}{l}\text { De } 2 \text { hojas, } 2 \text { de pomelo, } 2 \text { de durazno, } 2 \text { de níspero y ambay. Tomar con } \\
\text { una pastilla }\end{array}$ \\
\hline & & & Cocimiento & De 3-4 hojas por taza de agua \\
\hline & & \multirow{5}{*}{ Gripe } & \multirow{4}{*}{ Infusión } & De 2-5 hojas. Dejar entibiar y tomar por la noche \\
\hline & & & & $\begin{array}{l}\text { De } 2 \text { hojas, } 2 \text { de pomelo, } 2 \text { de durazno, } 2 \text { de níspero y ambay. Tomar con } \\
\text { una pastilla }\end{array}$ \\
\hline & & & & $\begin{array}{l}\text { De } 2 \text { hojas, } 2 \text { de mandarina y } 2 \text { hojas de salvia o de prontoalivio. Agregar } \\
\text { azúcar quemado con un carboncito }\end{array}$ \\
\hline & & & & $\begin{array}{l}\text { Con azúcar quemado y mezclado con brotes de talera. Se acompaña con una } \\
\text { novalgina o bayer }\end{array}$ \\
\hline & & & \multirow{3}{*}{ Cocimiento } & Con hojas de salvia y/o de ambay. Agregar azúcar y tomar con una aspirina \\
\hline & & Angina & & Dejar enfriar y hacer gárgaras \\
\hline & & Antiséptico & & De 200 gramos de hojas. Dejar enfriar \\
\hline & & \multirow{2}{*}{ Sedante } & \multirow{3}{*}{ Infusión } & 2-4 hojas por taza. Tomar por la noche \\
\hline & & & & De 6-9 hojas, con azúcar \\
\hline & & Laxante & & En agua o en leche \\
\hline & & $\begin{array}{l}\text { Dolores óseos y de } \\
\text { cintura }\end{array}$ & Cocimiento & Baños \\
\hline & & Digestivo & Infusión & De 2-3 hojas por taza. Tomar en ayunas \\
\hline & & Males del hígado & & \\
\hline & Jugo & $\begin{array}{l}\text { Enfermedades } \\
\text { respiratorias }\end{array}$ & Jarabe & Jugo disuelto en agua azucarada o miel. Se toma a cucharaditas, entibiado \\
\hline
\end{tabular}




\begin{tabular}{|c|c|c|c|c|}
\hline \multirow{10}{*}{ NARANJA } & \multirow{3}{*}{ Jugo } & Refrescante & & \multirow{2}{*}{ Bebido } \\
\hline & & Sedante & \multirow{2}{*}{ Jugo } & \\
\hline & & Digestivo & & Agregado en infusiones \\
\hline & \multirow{2}{*}{ Fruto } & Gripe & \multirow{3}{*}{ Cocimiento } & \multirow{2}{*}{ De la fruta cortada en 1 taza de agua. Agregar miel y bayaspirina } \\
\hline & & Fiebre & & \\
\hline & \multirow{4}{*}{ Cáscara } & & & Junto con miel. Tomar todas la noches \\
\hline & & Gripe & \multirow{4}{*}{ Infusión } & $\begin{array}{l}\text { Quemar (sapecar) la cáscara en el fuego y agregarle agua caliente. Tomar con } \\
\text { bayaspirina o geniol }\end{array}$ \\
\hline & & Resfrío & & Con agregado de jugo de limón y endulzado con azúcar o miel \\
\hline & & Para la tos & & Con hojas de salvia y jugo de pomelo \\
\hline & $\begin{array}{l}\text { Hojas y } \\
\text { epicarpo }\end{array}$ & Fiebre & & \\
\hline \multirow{14}{*}{ LIMÓN } & \multirow{14}{*}{ Jugo } & \multirow[b]{2}{*}{ Fiebre } & \multirow[b]{2}{*}{ Jugo } & Agregado a infusión de te o te negro con aspirina, tafirol, geniol o ibuprofeno \\
\hline & & & & $\begin{array}{l}\text { Agregado a infusiones de te negro, con cáscaras de naranja o pomelo, azúcar } \\
\text { quemado y geniol }\end{array}$ \\
\hline & & Refrescante & Jugo con agua y azúcar & Contraindicado para los jóvenes, por tener sangre fina \\
\hline & & \multirow{4}{*}{ Gripe } & Jugo & Agregado a la infusión de te negro con aspirina, tafirol o ibuprofeno \\
\hline & & & & Solo o con bayaspirina \\
\hline & & & Jarabe & Con miel de abeja. Entibiar \\
\hline & & & Infusión & Con geniol \\
\hline & & Angina & \multirow{5}{*}{ Jarabe } & Mezclado con 3 cucharadas de miel de abeja de monte \\
\hline & & Bronquitis & & Jugo mezclado con miel de yateí, o en su defecto de abeja \\
\hline & & Expectorante & & Con miel de abejas. Entibiar \\
\hline & & \multirow{3}{*}{ Para la tos } & & Jugo con azúcar. Tomar caliente \\
\hline & & & & Cocimiento del jugo con miel de yateí o de abeja \\
\hline & & & Cocimiento & $\begin{array}{l}\text { De } 1 \text { litro de jugo, } 1 \text { litro de vino tinto y un kilo de azúcar hasta reducir a } 1 \\
\text { litro total. Tomar } 2-3 \text { cucharadas por día }\end{array}$ \\
\hline & & Resfrío & Jugo & $\begin{array}{l}\text { Agregado a infusión de te con azúcar quemado con un carboncito. } \\
\text { Acompañar con un geniol }\end{array}$ \\
\hline
\end{tabular}




\begin{tabular}{|c|c|c|c|c|}
\hline \multirow{24}{*}{ LIMÓN } & \multirow{12}{*}{ Jugo } & Resfrío & Infusión & De cáscaras de naranja, endulzado con azúcar o miel \\
\hline & & Para no vomitar & \multirow{7}{*}{ Jugo } & Bebido \\
\hline & & Digestivo & & Agregado a infusiones o sopas \\
\hline & & Dolor de panza & & Remedio fresco \\
\hline & & Colitis & & \\
\hline & & Acidez & & \\
\hline & & Afinar la sangre & & Bebido \\
\hline & & Vesícula & & \\
\hline & & Aftas en la boca & \multirow[b]{2}{*}{ Tópico } & \multirow[b]{2}{*}{ Aplicar en la zona afectada } \\
\hline & & $\begin{array}{l}\text { Antiinflamatorio en } \\
\text { picaduras }\end{array}$ & & \\
\hline & & Preventivo & Jugo & Con agua \\
\hline & & Para adelgazar & Jugo & Con agua, cebado en tereré. Su abuso produce anemia \\
\hline & \multirow{7}{*}{ Fruto } & \multirow{4}{*}{ Gripe } & \multirow[b]{2}{*}{ Cocimiento } & De medio fruto en agua. Tomar con bayaspirina o geniol \\
\hline & & & & $\begin{array}{l}\text { Del fruto entero cortado en cuatro. También puede ser acompañado con } \\
\text { bayaspirina o paracetamol }\end{array}$ \\
\hline & & & Freido & Medio fruto con aceite vegetal, azúcar y miel \\
\hline & & & \multirow{4}{*}{ Cocimiento } & Del fruto mezclado con cedrón del campo y/o ambay \\
\hline & & Fiebre & & Del fruto entero cortado en cuatro. Tomar con bayaspirina o paracetamol \\
\hline & & Resfrío & & Del fruto mezclado con cedrón del campo y/o ambay \\
\hline & & Dolor de garganta & & Del fruto partido \\
\hline & Flores & \multirow{3}{*}{ Gripe } & Infusión & \\
\hline & \multirow{4}{*}{ Hojas } & & Cocimiento & $\begin{array}{l}\text { Con azúcar quemado y mezclado con brotes de talera. Se acompaña con } \\
\text { novalgina o bayer }\end{array}$ \\
\hline & & & Infusión & Con hojas de mandarina y ambay \\
\hline & & Para la tos & Cocimiento & $\begin{array}{l}\text { Con azúcar quemado y mezclado con brotes de talera. Se acompaña con } \\
\text { novalgina o bayer }\end{array}$ \\
\hline & & Bajar la presión & Infusión & \\
\hline LIMÓN REAL & Jugo & Refrescante & Jugo & Con agua y azúcar \\
\hline \multirow{2}{*}{ LIMÓN SUTIL } & \multirow{2}{*}{$?$} & Gripe & & \\
\hline & & Para la tos & & \\
\hline
\end{tabular}


Figura 2 - Fitoterapia local con cítricos. A. Dolencias tratadas con los cítricos (Los números representan la cantidad de menciones totales). B. Partes de la planta empleadas en la preparación de las recetas medicinales: porcentajes de menciones. C. Forma de preparación de las recetas medicinales mostrando los porcentajes de menciones para cada una.
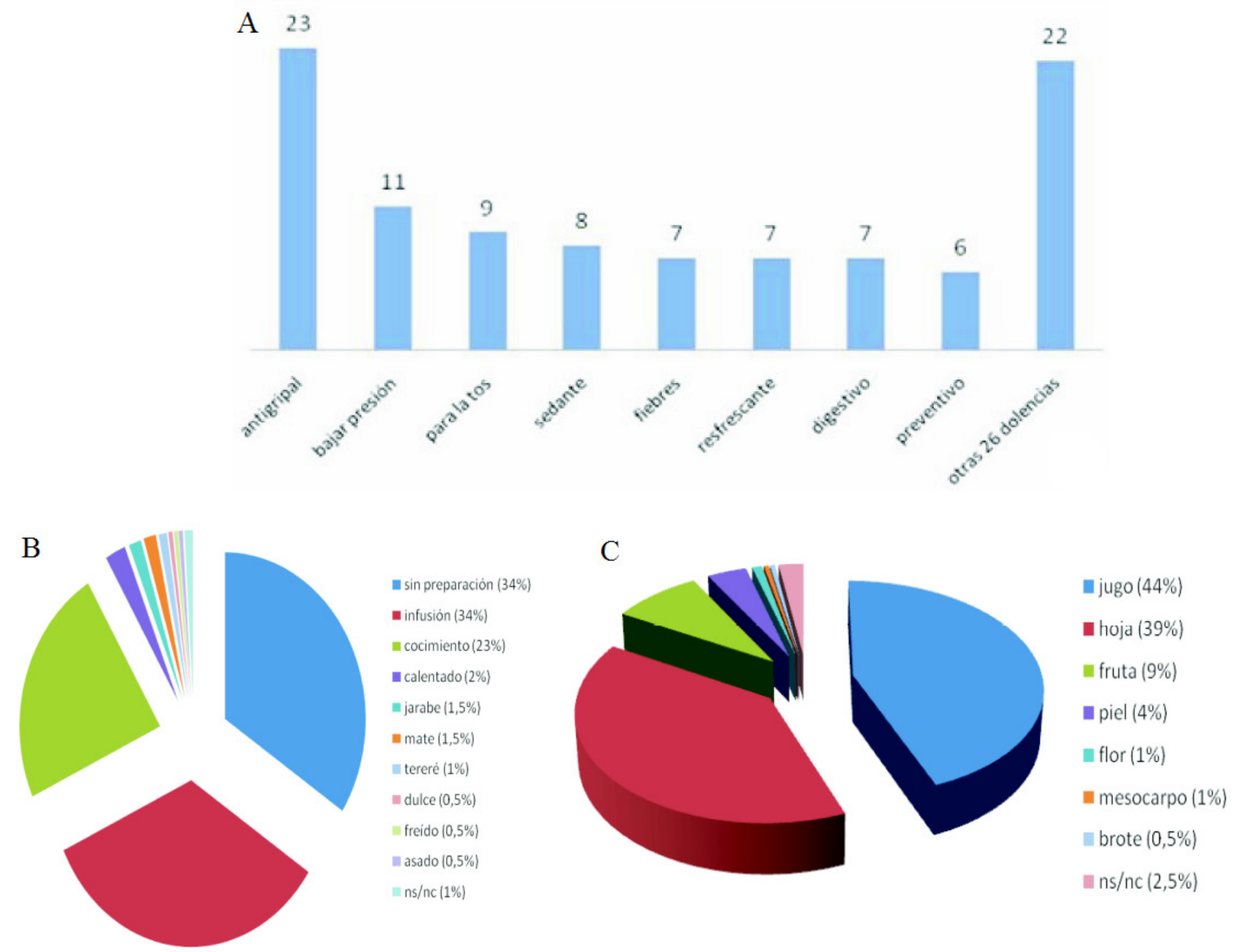

\section{Discusión}

De las 7 etnoespecies relevadas para el tratamiento de distintas dolencias, la "cidra" resultó ser la menos importante -una mención de uso actual-, coincidiendo con el desuso de este frutal a medida que los descendientes de los colonos instalados desde inicios del siglo XX van incorporándose al ámbito urbano. Asimismo, se encuentra ausente en este listado el "cidro" (C. medica), sin embargo, se emplea con el mismo fin el "limón cidra" (C. $\times$ limon), híbrido del cual C. medica es uno de los parentales. Esta etnoespecie es citada para el NE de Argentina y Paraguay con numerosas propiedades medicinales relacionadas al sistema digestivo, nervioso, en fiebres y como antihelmíntico (Hieronymus 1882; Rodríguez Barboza 1985). Sin embargo, queda para confirmar a futuro a que referían estos autores dada la similitud en los términos y usos con el "limón cidra" referido en este trabajo. Más recientemente, es citado en trabajos etnobotánicos para las yungas del noroeste argentino como tónico en preparaciones junto a otros cítricos (Hilgert 1999). Esta autora destaca la retracción de esta especie en el área de su estudio, por lo cual actualmente es común adquirir su fruto desecado en los mercados. La "lima ácida" (C. $\times$ aurantiifolia), escasamente representada en el sur de Misiones, está asimilada localmente a la etnoespecie 
"limón" debido a la acidez permanente del endocarpo, al igual que en otros enclaves de América (Vandebroek \& Balick 2014; Stampella et al. 2013; Hilgert et al. 2014), no así en el ámbito de la citricultura argentina (Palacios 1978).

Las dolencias registradas en el área que se tratan con cítricos coinciden en gran parte con la bibliografía. Sin embargo, el uso con fines refrescante tanto de las formas ácidas como de las dulces adquiere un alto valor en la zona, dadas las elevadas temperaturas estivales. Este uso posiciona a los cítricos dentro de los frutales locales más valiosos. Por esta razón los cítricos pueden ser considerados como alimentos funcionales, es decir plantas empleadas en la alimentación con propósitos terapéuticos, lo que remite, a la vez, a un concepto holístico y unicista de salud/enfermedad (Pochettino et al. 2012). En coincidencia y en este marco, algunos cítricos son mencionados como alimentos funcionales en diversas partes del mundo, tanto como por sus propiedades curativas como preventivas al evitar enfermedades relacionadas con deficiencias en determinados alimentos, como por ejemplo avitaminosis (Etkin 2008; Vandebroek \& Balick 2014).

Asimismo, el empleo del jugo de "limón" para afinar la sangre fue mencionado por un informante en la ciudad de San Ignacio. El mismo se refirió a esta dolencia como "la sangre se pone gruesa cuando uno come mucho frito, mucha grasa, entonces no corre por el cuerpo y uno se siente pesado...”. Esto mismo ha sido citado para España (Alto Aragón) por Palacín et al. (1984).

El pasmo merece mayor atención, debido a la disparidad de sus descripciones. Fue presentado como "una infección que hincha, es bien jodido" (SF., Cerro Mártires); también como "hinchazón, inflamación que viene por estar trabajando en el calor y pasar al frío o tomar agua fría. A veces pasa en los dientes y pareciera como que se te aflojaran o que se hincharan para infectarse" (E. V., Cerro Mártires). Estas definiciones se acercan más a las de Plath (1996: 251) y Thomas \& Vandebroek (2006), consistentes en inflamaciones con edemas y dolor localizado, aunque también puede tratarse de heridas infectadas (pasmadas), incluyendo la inflamación de las mejillas por infección dental, afección registrada también por Cadogan (1957) para el norte de Paraguay con el nombre de tétanos. Asimismo, es de destacar la importancia de la Teoría Humoral (frío-calor) relacionada con esta dolencia (Hilgert 2001; Scarpa 2012).

Se registró el uso de los cítricos en el tratamiento de la hipertensión, en coincidencia con menciones previas en la región, en los que el "pomelo", el "limón”, la "lima ácida” y la "apepú" fueron citados en otras regiones del NEA para el tratamiento de este padecimiento (Martínez-Crovetto 1981; Amat \& Yajía 1991; Keller \& Romero 2006), así como en estudios realizados en Paraguay y el sur de Brasil (Rodríguez Barboza 1985; Ritter et al. 2002; Vendruscolo \& Mentz 2006). Los estudios sobre las acciones farmacológicas de este género confirman que los frutos de "naranja", "limón", "mandarina", "pomelo" y "lima dulce" (C. $\times$ limon cv 'Limetta') tienen efecto hipotensor en animales de laboratorio (García Mesa et al. 2002; Leite et al. 2008; Pérez et al. 2010; Lopes Campêlo et al. 2011). Asimismo, se ha demostrado que los aceites esenciales presentes en casi toda la planta más concentrados en las flores, específicamente linalol, acetato de linalilo, nerol y geraniol- tienen propiedades sedantes, hipnóticas y antiespasmódicas, que repercuten en la presión arterial (Alonso 2004).

Con respecto a las dolencias tratadas la mayoría de las menciones se refieren a procesos gripales y febriles, luego respiratorios, digestivos y sanguíneos. La mayor parte de la bibliografía cita que estos sistemas son tratados con los cítricos (Hieronymus 1882; Martínez-Crovetto 1981; Keller \& Romero 2006). Los trabajos de Zamudio et al. (2010) y Kujawska et al. (2012) citan a las enfermedades respiratorias como las principales tratadas con los cítricos, quizá debido al acercamiento como plantas acompañantes de remedios a base de mieles, empleadas 
principalmente para dolencias relacionadas a este sistema.

Este elenco de etnoespecies relevadas es similar a las presentadas para el norte de la provincia en comunidades de criollos y polacos (Kujawska et al. 2012; Kujawska \& Hilgert 2014; Kujawska \& Pieroni 2015), incluso en las preparaciones medicinales a base de mieles de diversas especies de abejas (Zamudio et al. 2010). En estos trabajos los cítricos resultaron más importantes en las comunidades de criollos, presentando altos valores de importancia relativa y de menciones, especialmente el "limón mandarina" y la "naranja dulce". En los alrededores de la Reserva de Biósfera Yabotí (centro-este de Misiones), Keller \& Romero (2006), en cambio, citan a la "lima ácida", pero no a la "lima [dulce]" y el "pomelo". Así, estas particularidades locales, especialmente ricas en la provincia debido al "mosaico cultural" actual resultado de su historia, deben ser tenidas en cuenta en estudios prospectivos ya que determinadas etnoespecies están asociadas a diferentes vertientes culturales.

\section{ConClusión}

Los cítricos representan alimentos funcionales en el sentido de su uso cotidiano como frutal y para la preparación de diversos remedios para el tratamiento de distintas dolencias. A la vez, se trata de plantas clave en las comunidades pluriculturales del sur de Misiones ya que son empleadas como forraje para el ganado y cebos para caza y pesca (ver Riat et al., en este volumen).

Este amplio abanico de etnoespecies y partes empleadas, modos de preparación y dolencias tratadas muestra la importancia local de los cítricos en los conocimientos locales de los criollos de Misiones, resaltando la importancia cultural de los mismos, aspecto de relevancia en el momento de establecer estrategias de conservación in situ.

\section{Agradecimientos}

Queremos agradecer profundamente a las comunidades locales de Misiones. Se agradece a CONICET y UNLP por los fondos provenientes de los subsidios. Asimismo, se agradece al revisor anónimo por los aportes a este trabajo.

\section{REFERENCIAS}

Albuquerque UP, Cunha LVFC, Lucena RFP \& Alves RRN (Eds.). 2014. Methods and techniques in ethnobiology and ethnoecology, New York: Humana Press, 480 p.

Alexiades MN \& Laird SA. 2002. Estableciendo los fundamentos: relaciones equitativas de la investigación sobre biodiversidad. In: Laird SA (Ed.), Biodiversidad y conocimiento tradicional. Participación equitativa en práctica, Montevideo: Nordan Comunidad, p. 41-52.

Alonso J. 2004. Tratado de Fitofármacos y Nutracéuticos, Rosario: Editorial CORPUS, 1360 p.

Amat AG \& Yajía ME. 1991. Plantas medicinales y etnofarmacología en la provincia de Misiones (Argentina). Acta Farmacéutica Bonaerense, 10(3):153-159. 
Arias BA \& Ramón-Laca L. 2005. Pharmacological properties of citrus and their ancient and medieval uses in the Mediterranean region. Journal of Ethnopharmacology, 97(1):89-95.

Bertoni M. 1927. La civilización guaraní, Parte III, Etnografía. Conocimiento. Puerto Bertoni: Imprenta y Edición "Ex Sylvis", 531p.

Burgstaller C. 1985. La vuelta a los vegetales. 600 hierbas medicinales y sus propiedades curativas. 700 fórmulas útiles y su preparación en el hogar, Buenos Aires: Ed. Hachette S.A., 631 p.

Cadogan L. 1957. Apuntes de medicina popular guaireña, Publicación del Centro de Estudios Antropológicos del Paraguay, Asunción: Imprenta Nacional, 57 p.

Capparelli A, Hilgert NI, Ladio A, Lema VS, Llano C, Molares S, Pochettino ML \& Stampella P. 2011. Paisajes culturales de Argentina: Pasado y presente desde las perspectivas etnobotánica y paleoetnobotánica. Revista de la Asociación Argentina de Ecología de Paisajes, 2(2):67-79.

Cotton CM. 1998. Ethnobotany: Principles and applications, UK.: John Wiley \& Sons, 424 p.

Crivos M, Martínez MR, Pochettino ML, Remorini C, Sy A \& Teves L. 2007. Pathways as "signatures in landscape": towards an ethnography of mobility among the Mbya-Guarani (Northeastern Argentina). Journal of Ethnobiology and Ethnomedicine, 3:2. doi: 10.1186/1746-4269-3-2.

De Grenade R, Krueger R, Nabhan GP \& Olvera MC. 2014. Mission and modern Citrus species diversity of Baja California Peninsula oases. Economic Botany, 68(3):262-282.

Etkin NL. 2006. Medicines. An ethnopharmacology of food, Tucson: The University of Arizona Press. 307 p.

García Mesa M; Herrera DMA, Vilas MM, Alfonso CC, Carretero JH, Batista AD \& Montequini JF. 2002. Plantas cítricas en el tratamiento de enfermedades vasculares. Revista Cubana de Angiología y Cirugía Vascular, 3(2):39-46.

Gmitter FG Jr \& Hu X. 1990. The possible role of Yunnan, China, in the origin of contemporary citrus species (Rutaceae). Economic Botany, 44(2):267-277.

Granitto GM \& Sarandón SJ. 2002. Producción de caña de azúcar orgánica: el caso de la cooperativa la Unión de San Juan de la Sierra, Misiones, Argentina In: Sarandón SJ (Ed.), Agroecología. El camino hacia una agricultura sustentable, La Plata: Ediciones Científicas Americanas, p. 549-557.

Hieronymus J. 1882. Plantae diaphoricae florae argentinae. Boletín de la Academia Nacional de Ciencias en Córdoba, 4(3):199-598.

Hilgert NI, Lambaré, DA, Vignale ND, Stampella PC \& Pochettino ML. 2014. ¿Especies naturalizadas o antropizadas? Apropiación local y la construcción de saberes sobre los frutales introducidos en época histórica en el norte de Argentina. Revista Biodiversidad Neotropical, 4(2):69-87. 
Hilgert NI. 1999. Las plantas comestibles en una zona de las Yungas meridionales (Argentina). Anales del Jardín Botánico de Madrid, 57(1):117-138.

Hilgert NI. 2001. Plants used in home medicine in the Blanco River basin, in northwestern Argentina subtropical montane forests. Journal of Ethnopharmacology, 76:11-34.

Keller HA \& Romero HF. 2006. Plantas medicinales utilizadas por campesinos del área de influencia de la Reserva de Biósfera Yabotí (Misiones, Argentina). Bonplandia, 15(3-4):125-141.

Kujawska M \& Hilgert NI. 2014. Phytotherapy of Polish migrants in Misiones, Argentina: Legacy and acquired plant species. Journal of Ethnopharmacology, 153:810-830.

Kujawska M, Zamudio F \& Hilgert NI. 2012. Honey-based mixtures used in home medicine by nonindigenous population of Misiones, Argentina. Evidence-Based Complementary and Alternative Medicine,volume 2012. Article ID 579350, 15 pages. Doi: 10.1155/2012/579350.

Kujawska M \& Pieroni A. 2015. Plants used as food and medicine by Polish migrants in Misiones, Argentina. Ecology of Food and Nutrition, 54:255-279.

Leite M, Fassin Jr J, Baziloni EMF, Almeida RN, Mattei R \& Leite JR. 2008. Behavioral effects of essential oil of Citrus aurantium L. inhalation in rats. Revista Brasileira de Farmacognosia, 18(Supl.):661-666.

Lewis WH. 2010. Evaluating and protecting indigenous pharmacopeias and traditional knowledge. In: Pochettino et al. (Eds.), Tradiciones \& transformaciones en Etnobotánica, San Salvador de Jujuy: CYTED - Programa Iberoamericano Ciencia y Tecnología para el Desarrollo, p. 313-316.

Lopes Campêlo LM, Lima SG, Feitosa CM \& Freitas RM. 2011. Evaluation of central nervous system effects of Citrus limon essential oil in mice. Revista Brasileira de Farmacognosia, 21(4):668-673.

Martínez-Crovetto R. 1981. Las plantas utilizadas en medicina popular en el noroeste de Corrientes (República Argentina). Miscelanea, 69:1-139.

Palacín JM, Pérez LV \& Calvo C. 1984. Plantas usadas como hipotensoras en el Alto Aragón. Acta Biológica Montana, 4:483-496.

Palacios J. 1978. Citricultura Moderna, Buenos Aires: Ed. Hemisferio Sur. 409 p.

Pérez Y, Ferrer EJ, Alonso D, Amaro CAB \& Zamilpa A. 2010. Citrus limetta leaves extract antagonizes the hypertensive effect of angiotensin II. Journal of Ethnopharmacology, 128(3):611-614.

Plath O. 1996. Folclor médico chileno, Santiago de Chile: Ed. Grijalbo S.A., 308 p.

Pochettino ML, Puentes JP, Buet Constantino F, Arenas PM, Ulibarri E \& Hurrell JA. 2012. Functional foods and nutraceuticals in a market of Bolivian inmigrants in Buenos Aires (Argentina). Evidence-Based Complementary and Alternative Medicine, 2012, Article ID 320193, 14 pages. DOI: 10.1155/2012/320193.

Ramón-Laca L. 2003. The introduction of cultivated citrus to Europe via Northern Africa and the Iberian 
Peninsula. Economic Botany, 57(4):502-514.

Ritter MR, Sobierajski GR, Schenkel EP \& Metnz LA. 2002. Plantas usadas como medicinais no municipio de Ipê, RS, Brasil. Revista Brasileira de Farmacognosia, 12(2):51-62.

Rodolfo AM, Cândido Jr JF, Temponi LG \& Gregorini MZ. 2008. Citrus aurantium L. (laranja-apepu) e Hovenia dulcis Thunb. (uva-do-japão): espécies exóticas invasoras da trilha do Poço Preto no Parque Nacional do Iguaçu, Paraná, Brasil. Revista Brasileira de Biociências, 6(1):16-18.

Rodríguez Barboza N. 1985. Pojhá Ñaná. Recetario de plantas medicinales usadas en el Paraguay, Asunción: Ed. La Colmena, 356 p.

Scarpa GF. 2012. Las plantas en la vida de los criollos del oeste formoseño. Medicina, ganadería, alimentación y viviendas tradicionales, Buenos Aires: Asociación Civil Rumbo Sur, 237 p.

Stampella PC, Delucchi G, Keller HA \& Hurrell JA. 2014. Etnobotánica de Citrus reticulata (Rutaceae, Aurantioideae) naturalizada en la Argentina. Bonplandia, 23(2):151-162.

Stampella PC, Lambaré DA, Hilgert NI \& Pochettino ML. 2013. What the iberic conquest bequeated to us: the fruit trees introduced in argentine subtropic, their history and importance in present traditional medicine. EvidenceBased Complementary and Alternative Medicine,Article ID 868394, 17 pages. Doi: 10.1155/2013/868394.

Thomas E \& Vandebroek I. 2006. Guía de plantas medicinales de los Yuracarés y Trinitarios del Territorio Indígena Parque Nacional Isiboro-Sécure, Bolivia, Santa Cruz: Imprenta Sirena, 522 p.

Vandebroek I \& Balick MJ. 2014. Lime for chest congestion, bitter orange for diabetes: foods as medicines in the Dominican community in New York City. Economic Botany, 68(2):177-189.

Vendruscolo G \& Mentz LA. 2006. Levantamento etnobotânico das plantas utilizadas como medicinais por moradores do bairro Ponta Grossa, Porto Alegre, Rio Grande do Sul, Brasil. Iheringia, Sér. Bot., 61(1-2):83103.

Webber H. 1943. History and development of the Citrus industry. In: Webber H \& Batchelor L (Eds.), The Citrus Industry. History, Botany, and Breeding, Berkeley: University of California Press, p. 1-40.

Zamudio F, Kujawska M \& Hilgert NI. 2010. Honey as medicinal and food resource. Comparison between Polish and multiethnic settlements of the Atlantic Forest, Misiones, Argentina. The Open Complementary Medicine Journal, 2:58-73.

Zamudio F \& Hilgert NI. 2011. Mieles y plantas en la medicina criolla del norte de Misiones, Argentina. Bonplandia, 20:165-184.

Zamudio F \& Hilgert NI. 2012. ¿Cómo los conocimientos locales aportan información sobre la riqueza de especies de abejas sin aguijón (Apidae: Meliponini) del norte de Misiones, Argentina? Interciencia, 37(1):36-43. 